\title{
Antitumor Immunity from Abdominal Flap-Embedded Therapeutic Cancer Vaccine
}

\author{
Xiaoling Liu ${ }^{1,2, *}$ \\ Junhua Mai (D)* \\ Chaoyang Meng' \\ Aldona J Spiegel ${ }^{3}$ \\ Wei $\mathrm{Wei}^{2}$ \\ Haifa Shen'
}

'Department of Nanomedicine, Houston Methodist Academic Institute, Houston, TX, 77030, USA; ${ }^{2}$ Department of Breast and Thyroid Surgery, Peking University Shenzhen Hospital, Shenzhen,

Guangdong, 518036, People's Republic of China; ${ }^{3}$ Institute for Reconstructive Surgery, Houston Methodist Hospital, Weill Cornell Medicine, Houston, TX, USA

*These authors contributed equally to this work
Correspondence: Haifa Shen

Department of Nanomedicine, Houston Methodist Academic Institute, Houston, TX, 77030, USA

Tel + I 7|3-44I-732I

Email HaifaShen@gmail.com
Background: Abdominal flaps are routinely performed in clinic after primary mastectomy of breast cancer. However, cancer patients can still develop cancer recurrence and metastasis after surgery. In this study, we evaluated the feasibility of concurrent abdominal flap reconstruction and vaccine inoculation in the tissue for prevention and treatment of HER2-positive breast cancer.

Methods: A murine model of metastatic HER2-positive breast cancer was generated by inoculating HER2-expressing TUBO tumor cells into both the mammary gland fat pad and left ventricle. Mammary gland fat pad with primary tumor was resected by mastectomy, and superficial inferior epigastric (SIE) vessel-based abdominal flap was performed for abdominal reconstruction. During the surgery, mice also received a single intra-flap treatment of a microparticulate-based cancer vaccine. Popliteal (Pop) and inguinal (Ing) lymph nodes (LN) were collected at different time points after vaccination, and activation of dendritic cells and T lymphocytes was evaluated with flow cytometry. ELISpot was also performed to measure HER2-specific $\mathrm{T}$ cells in splenocytes. In addition, infiltration of $\mathrm{CD}^{+} \mathrm{T}$ cells in brain metastatic nodules was analyzed with immunohistochemistry.

Results: Flow cytometry detected increased number of activated dendritic cells in lymph nodes in mice treated with cancer vaccine. ELISpot revealed abundant IFN- $\gamma$-expressing $\mathrm{T}$ cells in the spleen. Mice treated with abdominal flap-embedded cancer vaccine extended median survival by 9 days over the control group $(\mathrm{p}<0.05)$.

Conclusion: Abdominal flap-embedded cancer vaccine effectively stimulated systemic immune response and inhibited tumor progression in a murine model of HER2-positive breast cancer.

Keywords: silicon microparticle, cancer vaccine, peddled flap-based reconstruction, breast cancer, HER2

\section{Introduction}

Breast cancer had surpassed lung cancer as the major cause of cancer death of women in the United States in 2019. ${ }^{1}$ It is classified by the expression of estrogen receptor (ER), progesterone receptor (PR) and human epidermal growth factor receptor 2 (HER2), as well as claudin. HER2 amplification occurs in $15-25 \%$ of patients with primary breast cancer, and HER2-positive breast cancer is considered intrinsically aggressive. ${ }^{2,3}$ With the availability of monoclonal antibodies (mAbs), such as trastuzumab and pertuzumab, dramatic improvement has been achieved in clinic for HER2-positive breast cancer therapy. However, resistance to $\mathrm{mAb}$ therapy and relapse of disease still pose grave challenges. ${ }^{4-6}$

Lumpectomy followed by whole breast radiation therapy is the first line choice for early stage breast cancer. Total mastectomy followed by either immediate or 
delayed breast reconstruction is also a surgical option for patients who are not suitable for breast conserving surgery with a better cosmetic appearance. There are multiple methods for breast reconstruction, and autologous reconstruction with tissues from abdominal tissue-based pedicled transverse rectus abdominal muscle (TRAM) flap, the free TRAM flap, or deep inferior epigastric perforator (DIEP) flap is frequently performed. Unfortunately, although the overall prognosis of breast cancer is favorable, a notable percentage of patients eventually develop local recurrence, especially in HER2-positive and triplenegative subtypes. About $3.9 \%$ of early stage breast cancer patients suffered from loco-regional recurrence (LRR) after a 5-year follow-up in a large analysis including 4841 patients who underwent mastectomy. ${ }^{1,7}$ The impairment of immunity by surgery and general anesthesia was a major reason of cancer recurrence and metastases. ${ }^{8}$

Immune therapy is an effective strategy for cancer treatment, and has achieved great success in recent years. Several immune checkpoint inhibitors targeting PD-1, PDL1 and CTLA-4 have been applied for breast cancer treatment, including triple-negative breast cancer (TNBC). ${ }^{9,10}$ Besides, chimeric antigen receptor (CAR)-T cell therapy for hematological malignancies, and dendritic cell-based therapeutic vaccine Sipuleucel- $T$ for prostate cancer patients have been approved by FDA. ${ }^{11,12}$ Although immune therapy has shown a broad application potential, inflamed tumors with high mutational burdens, high DNA microsatellite instability, abundant tumor infiltrating lymphocytes (TILs) and high expression of checkpoint blockade molecules have more favorable prognosis after immune therapy. Overall, breast cancer has been considered as a non- to moderately-inflamed cancer type, and TNBC and HER2-positive breast cancer (HER2+ BC) have higher immunogenic potential than the other subtypes. ${ }^{13,14}$ In the case of HER2+ BC, it has been shown that the cancer tissues have high levels of TILs, PD-L1 expression and DNA mutation burden. ${ }^{15,16}$ As the gold standard treatment strategy for HER2 + BC, trastuzumab and pertuzumab have demonstrated superb therapeutic benefit, and the outcome is partially from the host immune response. ${ }^{17}$ Nelipepimut-S, a peptide vaccine composed of an E75 HER2 peptide (KIFGSLAFL) and a GM-CSF adjuvant, can induce anti-tumor immune responses in HER2+ BC patients. ${ }^{18}$

Structurally, adipose tissue occupies most of the space in breast and forms mammary gland fat pad. The tissues not only serve as an energy depot, but also produce a unique microenvironment by recruiting immune cells and secreting cytokines. Dendritic cells (DCs) in the fat tissue can take up antigens from collecting lymphatic vessels, and then transport them to draining lymph nodes where they are presented to naive T cells. ${ }^{19}$ In addition, large adipocytes can function as non-professional antigen-presenting cells. ${ }^{20}$ Interestingly, both DCs and adipocytes express Toll-like receptor 9 (TLR9). ${ }^{21}$ TLR9 mediates strong NF-kb and type I interferon (IFN-I) response by activating the myeloid differentiation primary response 88 (MyD88) pathway. ${ }^{22}$ TLR9 agonists can therefore serve as an immune activator in adipose tissue. To take advantage of the potential immune environment of adipose flap tissue, local application of immune therapy on flap after breast reconstruction surgery may provide a favorable anti-cancer immunity to protect patients from tumor relapse and metastasis. In previous studies, we have developed a particulate therapeutic cancer vaccine platform by packaging soluble adjuvants and antigen peptides into micro-porous silicon microparticles (PSMs). The particulate vaccines are able to stimulate IFN-I response in DCs, resulting in potent anti-cancer immunity. ${ }^{23,24}$

In this study, we examined anti-cancer immune responses after inoculating an HER2-specific particulate vaccine in abdominal flap. The vaccine is composed of nanoporous silicon microparticles loaded with $\mathrm{CpG}$ oligodeoxynucleotide and HER2-specific antigen peptide ( $\mu$ CHER2). Both systemic and local immune responses were examined. $\mu$ CHER 2 activated DCs and induced strong antigen-specific $\mathrm{T}$ cell response. In addition, $\mu$ CHER 2 demonstrated efficacy in preventing local recurrence and inhibiting distal metastasis in a murine model of HER2-positive breast cancer.

\section{Materials and Methods Mice}

All animal experiments were performed according to the National Institutes of Health Guide for the Care and Use of Laboratory Animals and approved by Institutional Animal Care and Use Committee (IACUC) of Houston Methodist Research Institute. Six- to eight-week-old female Balb/c mice were purchased from Charles River Laboratories (Boston, MA, USA). All mice were housed in the animal facilities at the institute with conditional temperature, humidity and photoperiods (12-hour light/ 12-hour dark cycles).

\section{Cells and Cell Culture}

Murine breast cancer cell line TUBO that expresses a rat HER 2 gene was kindly provided by Dr. Zen Wei (Wayne 
State University, Detroit, MI) and Guido Forni (University of Turin, Italy). TUBO cells were engineered with a luciferase reporter gene. Cells were cultured in DMEM medium supplemented with $10 \%$ fetal bovine serum (FBS) and 100 units $/ \mathrm{mL}$ penicillin $-100 \mu \mathrm{g} / \mathrm{mL}$ streptomycin at $37{ }^{\circ} \mathrm{C}$ with $5 \% \mathrm{CO}_{2}$ and $95 \%$ humidity.

\section{Preparation of Particulate Vaccine}

Manufacture of silicon-based microparticles and preparation of particulate vaccine were processed as previously described. ${ }^{23}$ Briefly, $100 \mu \mathrm{g}$ p66 HER2 antigen peptide (TYVPANASL, synthesized by Peptide $2.0 \mathrm{Inc}$ ) and 10 $\mu \mathrm{g}$ CpG (ODN1826, Invivogen) were dissolved in water, and mixed with $20 \mathrm{mg} / \mathrm{mL}$ 1,2-dioleoyl-sn-glycero3-phosphocholine (DOPC, Avanti Polar Lipids) and 0.1\% Tween-20, followed by freezing and lyophilization. The lyophilized sample was rehydrated with water, and then loaded into 0.6 billion $1 \mu \mathrm{m}$ nanoporous silicon microparticles via sonication. To monitor biodistribution and particle transport, $0.5 \%$ (mol\%) 1,2-dioleoyl-sn-glycero3-phosphoethanolamine-N-(Cyanine 5.5) (Cy5.5-DOPE, Avanti Polar Lipids) was added to prepare fluorescent vaccine particles. Zeta potential and size measurements were carried out using a Zeta Sizer Nano ZS (Malvern Instruments, UK).

\section{Transport of $\mu \mathrm{CHER} 2$ Vaccine}

Transport of vaccine particles was examined by monitoring fluorescence of Cy5.5 labeled fluorescent vaccine in tissues with an IVIS-200 imaging system (Xenogen). Briefly, 0.2 billion Cy5.5 labeled $\mu$ CHER2 particles were administered in the fat pad (right 4th mammary fat pad). Mice were euthanized 24 hours after vaccination. Ipsilateral (right) and contralateral (left) Pop and Ing LNs, fat pad with vaccine inoculation, liver and spleen were collected for ex vivo imaging (em 675nm, ex 720nm).

\section{Evaluation of Immune Response After Vaccination}

Flow cytometry was applied to analyze immune cells in response to vaccination. Briefly, either 0.6 billion $\mu$ CHER2 particles or PBS control were injected into mammary gland fat pad of Balb/c mice. On the first day (leukocyte infiltration and DC activation) or the 7th day (T cell response) post-vaccination, spleen, ipsilateral Ing LNs and Pop LNs, and vaccinated fat pad were collected to prepare single cell suspension. DCs $\left(\mathrm{CD} 11 \mathrm{c}^{+} \mathrm{MHCII}{ }^{\mathrm{hi}}\right)$, myeloid cells $\left(\mathrm{CD} 11 \mathrm{~b}^{+}\right)$, neutrophils/
PMN-MDSCs $\quad\left(\mathrm{Ly}_{6 \mathrm{C}^{\text {int }}} \mathrm{Ly}_{6 \mathrm{G}^{+}}\right), \quad$ monocytes/M-MDSCs (Ly6 $\left.\mathrm{C}^{+} \mathrm{Ly}_{6 \mathrm{G}^{-}}\right), \quad \mathrm{T}$ cells $\left(\mathrm{CD}^{+}\right)$and $\mathrm{CD}^{+} \mathrm{T}$ cells $\left(\mathrm{CD}^{+}{ }^{+} \mathrm{CD} 8^{+}\right)$populations in different tissues were analyzed. All antibodies were purchased from Biolegend and $\mathrm{BD}$ Bioscience. To estimate antigen-specific T cells, p66 pentamer (ProImmune) was incubated with cell samples at room temperature, followed by staining of cell surface markers.

\section{ELISPOT Assay on T Cell IFN- $\gamma$}

To analyze activated splenocytes after vaccination, mice were immunized once according to the protocol mentioned above and then euthanized seven days after vaccination. Spleen, fat and lymph node samples were collected and processed into single cell suspension. Next, $2 \times 10^{5}$ cells/ well cells were inoculated into a pre-anti-IFN- $\gamma$-coated MultiScreen-IP plate (Millipore), and stimulated with 10 $\mu \mathrm{g} / \mathrm{mL}$ p66 peptide. Cells were then cultured for 36 hours. The plates were washed after incubation, followed by staining with biotinylated anti-IFN- $\gamma$ antibody and avidin-HRP (EBioscience). Spots were developed by adding AEC chromogen substrate.

\section{FLAP Surgery and Vaccination}

To generate mice with primary HER2-positive breast cancer, $2 \times 10^{5}$ TUBO cells were injected in the mammary gland fat pads of female Balb/c mice to produce orthotopic breast cancer. To generate mice with metastatic tumors, $1 \times 10^{5}$ TUBO cells were injected into left cardiac ventricle. Tumor growth was monitored with an IVIS-200 imaging system. On day 4 post-tumor inoculation, mice were randomly grouped, and orthotopic tumor resection and flap surgery were performed as described. ${ }^{25}$ All mice went through two steps of surgical procedure: mammary fat pad mastectomy and right superficial inferior epigastric (SIE) pedicel-based abdominal reconstruction.

Mastectomy included removal of the nipple-areola complex skin and tumor bearing breast fat pad. An abdominocutaneous flap based on the right SIE vessels containing whole thickness of skin and subcutaneous fat was elevated using fine dissecting scissors, and all perforators and lateral branches to the flap were disconnected. Entire elevated flap has the only SIE pedicel blood supply and venous drainage.

Vaccination was performed after the flap was prepared. In the control group, $50 \mu \mathrm{L}$ PBS was injected into the right 4th mammary fat pad. In the fat pad vaccination group, 0.6 billion vaccine particles were injected into the right 4 th mammary fat pad. After vaccination, the flap was re- 
sutured back to the skin lesion using 5-0 suture or skin clip. All flaps survived within 2 weeks post-surgery.

\section{Statistical Analysis}

Statistical significance between two test groups was analyzed using Student's $t$-test. One-way ANOVA was applied for the comparison among multiple groups. Animal survival was presented by Kaplan-Meier plotting, and evaluated using log rank test. P-values of less than 0.05 and 0.01 were considered statistically significant and very significant, respectively. Data are presented as means \pm SD.

\section{Results}

\section{Vaccination of $\mu \mathrm{CHER} 2$ Particle via Mammary Gland Fat Pad Injection}

To test the feasibility of intra-flap vaccination during beast reconstruction surgery, we prepared $\mu$ CHER2, and treated mice with intra-mammary gland fat pad injection. The overall procedure is shown in a schematic figure (Figure 1A). The $\mu$-particles were manufactured using electrochemical etching and photolithography, followed by APTES coating which provided a positively charged mesoporous surface (Figure 1A). ${ }^{26}$ We measured the PSM size with scanning electron microscope (SEM). As shown in Figure 1A, PSMs are $1 \mu \mathrm{m}$ in diameter and $400 \mathrm{~nm}$ in height, and with 40$100 \mathrm{~nm}$ pores. APTES modification led to slightly positive charge $(+2$ to $+4 \mathrm{mV})$ on the particle surface.
Surface modification protects particles from degradation and facilitates encapsulation of nanoparticles with antigen and adjuvants. Rat HER2-specific peptide p66 and the TLR9 agonist CpG were co-encapsulated in DOPC liposomes, which were about $68 \mathrm{~nm}$ in diameter and had a slightly negative surface charge of $-6.7 \mathrm{mV}$. Liposomes were then loaded into PSM with brief sonication to form $\mu$ CHER2, according to previous established protocols. ${ }^{24}$ For vaccination, Balb/c mice received injection of one dose of $50 \mu \mathrm{L} \mu \mathrm{CHER} 2$ on fat pad; in vivo and ex vivo imaging with an IVIS-200 system were then applied to track the particle migration. From

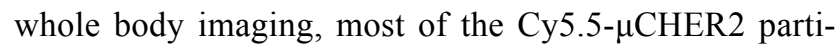
cles were stuck in original injection site at 24 hours post-vaccination (Figure 1A). To further investigate the biodistribution of Cy5.5- $\mu$ CHER2 particles and identify the major draining lymphatic tissue of the vaccination, fat pad, liver, spleen and lymph nodes were isolated for ex vivo imaging evaluation (Figure 1B). As expected, most of the injected particles were stuck in the original injection site, and a small portion was retained in liver probably by the mononuclear phagocyte system. Significant amount of Cy5.5- $\mu$ CHER2 particles accumulated in ipsilateral inguinal lymph nodes, which is considered as the major draining lymph node of the vaccine, as well as the place where antigen presentation and $\mathrm{T}$ cell priming happened. Vaccine particles in spleen and other lymph nodes were very limited. Based on the imaging result, therefore, vaccinated fat pad and its
A

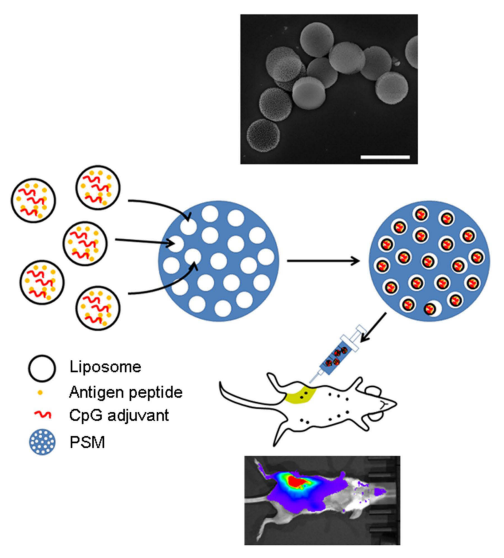

B

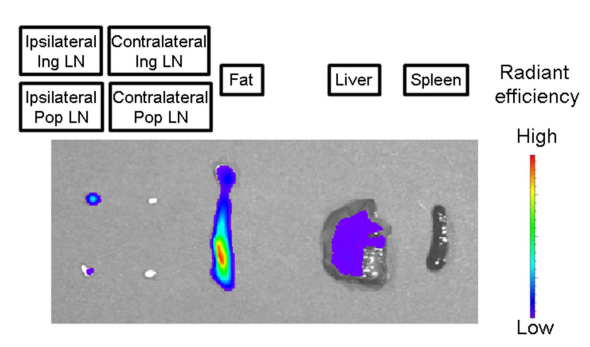

C

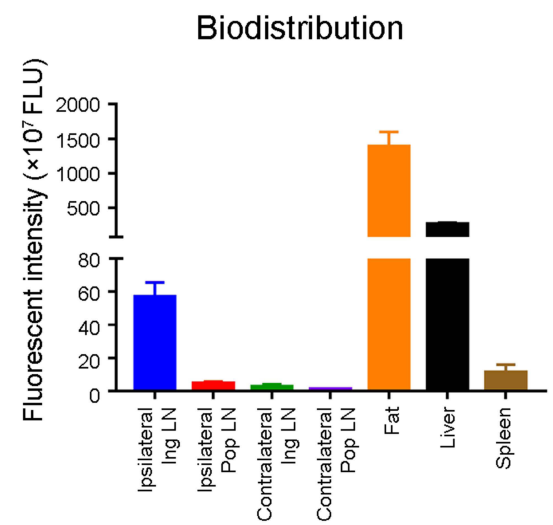

Figure I Intra-mammary gland fat pad injection of particular $\mu$ CHER2 vaccine. (A) Schematic figure of vaccine preparation and intra-mammary gland fat pad injection. Porous silicon particles were visualized with scanning electronic microscope. Bar indicates $2 \mu \mathrm{m}$. Whole body imaging was taken 24 hours after vaccination by in vivo imaging system (IVIS-200) to confirm the success of vaccine injection. (B) Tracking of $\mu$ CHER2 vaccine transport with IVIS-200 and (C) quantification of fluorescence intensity. Vaccine particles were prepared with Cy5.5-DOPE lipid, and dosed to mammary gland fat pad of Balb/c mice $(n=3)$. At 24 hours post-treatment, mice were euthanized to collect fat pad, lymph nodes and major organs for quantitative analysis of accumulation of vaccine particles. Data were presented as mean \pm SD. 
ipsilateral inguinal lymph nodes were further investigated for immune response (Figure 1C).

\section{Leukocyte Infiltration in Fat Tissue}

By vaccination via mammary gland fat pad, $\mu$ CHER2 was expected to stimulate immune response locally. To evaluate the pattern of fat immunization, we collected the vaccinated fat tissues for histological and cell-based analysis. By hematoxylin and eosin staining on fat pad sections, dramatic leukocyte infiltration was found in fat pad, both vessel adjacent area and around adipocytes 1 day after vaccination (Figure 2A). Most of the particulate vaccine stayed at the injection site, but some particulates had been taken up by immune cells and transported by the cells. To identify the subpopulations on infiltrating leukocytes, fat pad tissues were chopped and digested by collagenase, stained, and analyzed by flow cytometry. Overall, the amount of immune cells $\left(\mathrm{CD} 45^{+}\right)$was doubled after vaccination, indicating a strong recruitment of leukocytes by vaccine-inducted chemokines (Figure 2B).
The t-distributed stochastic neighbor embedding (t-SNE) analysis was performed to visualize the changes of cell populations with or without immunization (Figure 2C). After vaccination, the majority of monocytes $\left(\mathrm{CD} 11 \mathrm{~b}^{+} \mathrm{Ly} 6 \mathrm{C}^{+} \mathrm{Ly} 6 \mathrm{G}^{-}\right)$ showed a Ly6 $\mathrm{C}^{\text {hi }} \mathrm{MHC} \mathrm{II}^{-/ \text {int }}$ phenotype (Figure 2D), which was reported to be newly infiltrated from blood to inflammatory tissues. They may further differentiate into inflammatory macrophages and tumor necrosis factor- $\alpha$ and inducible nitric oxide synthase-producing dendritic cells (TIP-DCs) to promote local immunity. ${ }^{27}$ On the other hand, a group of

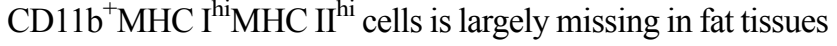
after vaccination. Lymphocytes $\left(\mathrm{CD} 45^{+} \mathrm{FSC}^{\mathrm{lo}} \mathrm{SSC}^{\mathrm{lo}}\right)$ and dendritic cells $\left(\mathrm{CD} 11 \mathrm{c}^{+} \mathrm{MHCII}{ }^{\mathrm{hi}}\right)$ are also mildly reduced. Neutrophils $\left(\mathrm{CD} 11 \mathrm{~b}^{+} \mathrm{Ly}_{6 \mathrm{C}^{\text {int }}} \mathrm{Ly}_{6 \mathrm{G}^{+}}\right)$and monocytes $\left(\mathrm{CD} 11 \mathrm{~b}^{+} \mathrm{Ly} \mathrm{C}^{+} \mathrm{Ly}_{6 \mathrm{G}}{ }^{-}\right)$were obviously recruited into vaccinated fat pad, showed a strong inflammatory response and leukocyte recruitment induced by vaccination (Figure $2 \mathrm{E}-\mathrm{G}$ ). Infiltrating monocytes and neutrophils reduced after 7 days (Figure 2H).
A

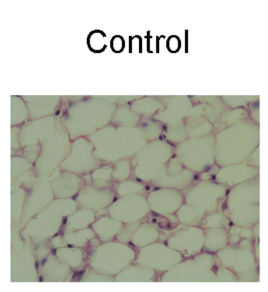

$\mu \mathrm{CHER} 2$
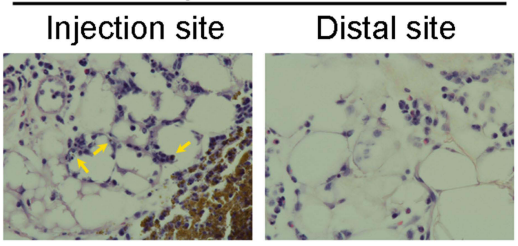

B
C

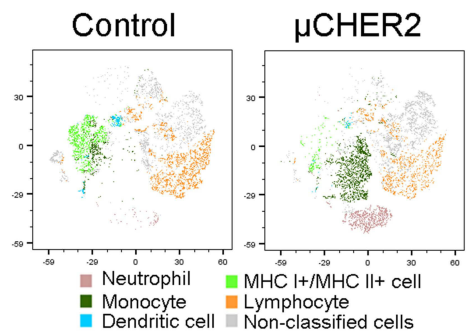

F
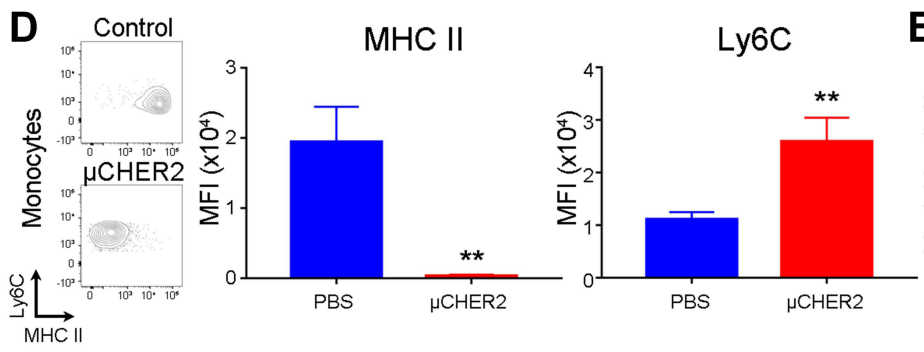

E
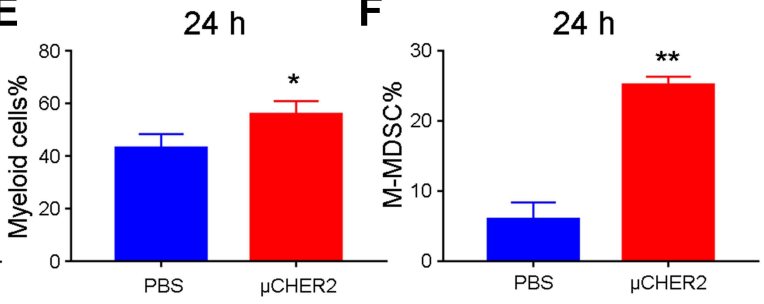

$72 \mathrm{~h}$

$72 \mathrm{~h}$
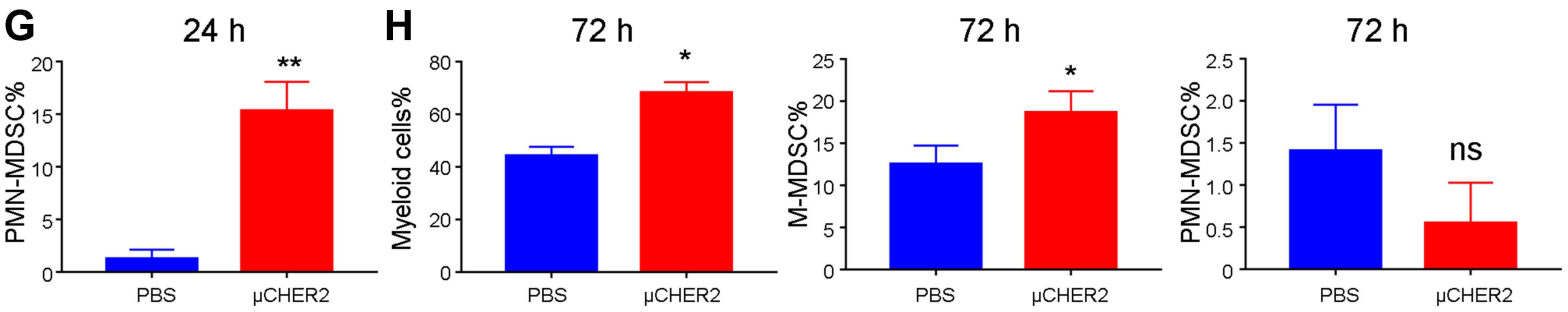

Figure 2 Infiltration of immune cells after fat pad vaccination. (A) Histological analysis of mammary gland fat pad at I day post-vaccine treatment. Fat pad samples were fixed and embedded in paraffin. Slices were stained with hematoxylin and eosin. $\mu$ CHER2 particles in migrating cells re indicated with yellow arrows. (B) Flow cytometry analysis on infiltrating leukocytes in fat pad 24 hours after vaccination ( $n=7$ mice per group). Different cell populations were analyzed at 24 hours by tSNE analysis (C). Total myeloid cells, monocytes and neutrophils were subsequently investigated at day I (D-G) or day 7 (H) samples in response to $\mu$ CHER2 treatment. Data were presented as mean \pm SD. ${ }^{*} \mathrm{p}<0.05,{ }^{* *} \mathrm{p}<0.01$ 


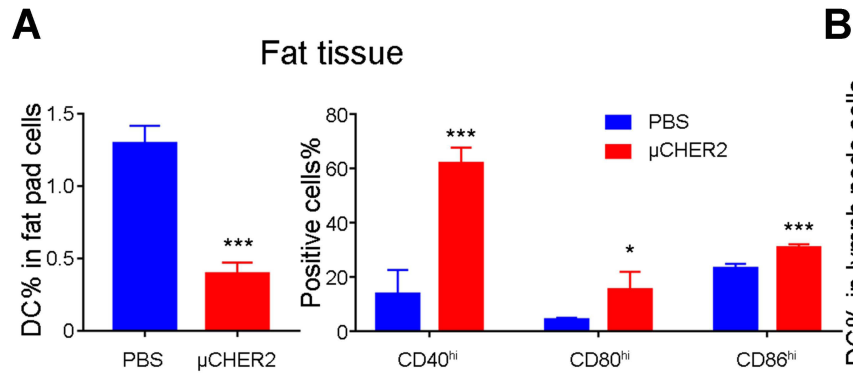

B

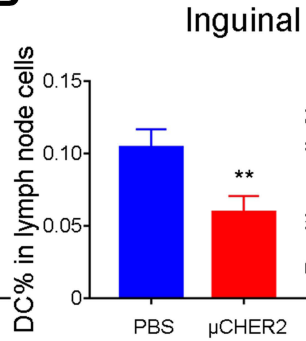

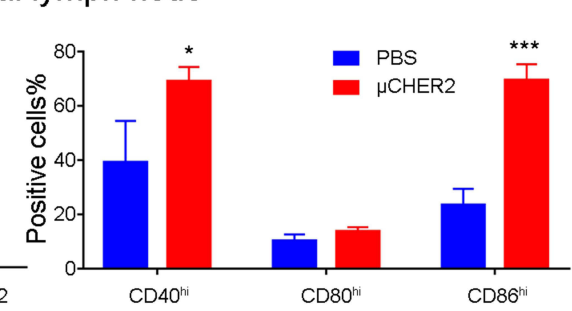

Figure 3 Analysis of activation and transport of dendritic cells after vaccination. At 24 hours post-vaccination, the population and activation of CDI Ic+MHC Ilhi DC in (A) fat pad and (B) ipsilateral Ing LNs were analyzed with flow cytometry. Data were presented as mean \pm SD. ${ }^{*} p<0.05, * * p<0.0$ I, $* * * p<0.00$ I.

\section{Activation by $\mu$ CHER2}

Dendritic cells played a key role in adaptive anti-cancer immunity. Our immune strategy is determined by the amount of dendritic cell activities triggered by the fat pad vaccination. We focused on the maturation and activation of dendritic cells after vaccination. We evaluated expression levels of CD40, CD80 and CD86 on DCs using flow cytometry. As mentioned above, a large amount of monocytes and neutrophils infiltrated from blood into inflammatory fat pad at 24 hours post-vaccination, thus DC percentage related to monocytes and neutrophils decreased (Figure 3A). The maturation of DCs is correlated to high elevation of costimulatory markers like CD40, CD80 and CD86, where their expressions on immature DCs stay at basal levels. At 24 hours post-vaccination, the majority of DCs in fat pad are CD $40^{\text {hi }}$ DCs as expected, whereas the CD80 ${ }^{\text {hi }}$ and CD86 ${ }^{\text {hi }}$ DC subsets are only slightly increased (Figure 3B). Mature DCs migrate to draining lymph nodes to prime $\mathrm{T}$ cells. We also investigated the DCs in inguinal lymph nodes, which is proved to be the draining lymph node of intra-fat pad injection (Figure 1B). DC population in inguinal lymph node moderately decreased (Figure 3C); however, predominant DCs were $\mathrm{CD} 40^{\mathrm{hi}} \mathrm{CD} 86^{\mathrm{hi}}$ cells. CD80 expression stayed at basal level.

\section{Antigen-Specific T Cell Response from $\mu$ CHER2 Treatment via Fat Pad}

Based on the DC activation results above, we expected to see that antigen-specific $\mathrm{T}$ cells can be primed by DCs activated by $\mu \mathrm{CHER} 2$, and proliferated. Consequently, we immunized the mice with $\mu$ CHER 2 via fat pad, and collected lymph nodes, spleen and fat tissues for flow cytometry measurement and enzyme-linked immune absorbent spot (ELISPOT) assay on IFN- $\gamma$. Tests were repeated and data were pooled for analysis. After vaccination, percentage of total $\mathrm{T}$ cells $\left(\mathrm{CD}^{+}\right)$and $\mathrm{CD}^{+} \mathrm{T}$ cells $\left(\mathrm{CD}^{+} \mathrm{CD}^{+}\right)$ remained almost the same (Figure 4A). Interestingly, significant amount of IFN- $\gamma$ producing $\mathrm{T}$ cells can be detected in splenocytes and inguinal lymph node cells. In contrast, as non-draining lymph node, IFN- $\gamma^{+}$cells were hardly detected. The antigen-specific $\mathrm{T}$ cells accumulated in fat pad, where breast cancer and its recurrence occurred. Via direct vaccination on fat, abundant IFN- $\gamma^{+}$cells were found (Figure 4B). HER2 pentamer specifically bound to HER2 specific CD $8^{+}$ $\mathrm{T}$ cells. In line with ELISPOT result in fat tissue, HER2 antigen specific $\mathrm{CD} 8^{+} \mathrm{T}$ cells dramatically increased and accumulated in fat pad where the vaccination took place. However, its percentage in spleen is low, which may indicate an insufficient systemic immunity (Figure 4C).

\section{Efficacy Study of Mastectomy and Flap Surgery Combined with Intra-Mammary Gland Fat Pad Vaccination}

Although mastectomy surgery aims to eliminate all tumor tissues, local or distant recurrence still sometimes occurs. To test whether recurrence can be prevented via $\mu$ CHER 2 treatment during surgery, we tested therapeutic efficacy on TUBO tumor model, in which mice bear both orthotopic and metastatic tumor. Surgery procedures are shown in Figure 5A, and were described previously. Most orthotopic tumors were removed by mastectomy followed by flap surgery, followed by $\mu$ CHER2 injection via fat pad (Figure 5B). The progression of systemic metastases and the development of local recurrence were monitored with an in vivo imaging system (Figure 5C). Surgery successfully removed most of the primary tumors; residual tumor cells were under the detection limit of current imaging technique, but had no effect on metastatic nodules. After vaccination in flap, anti-cancer immunity can only partially attenuate the growth of metastases in distant organs. However, we found that all five mice in the PBS group rapidly developed local recurrence; in contrast, only one of 
A
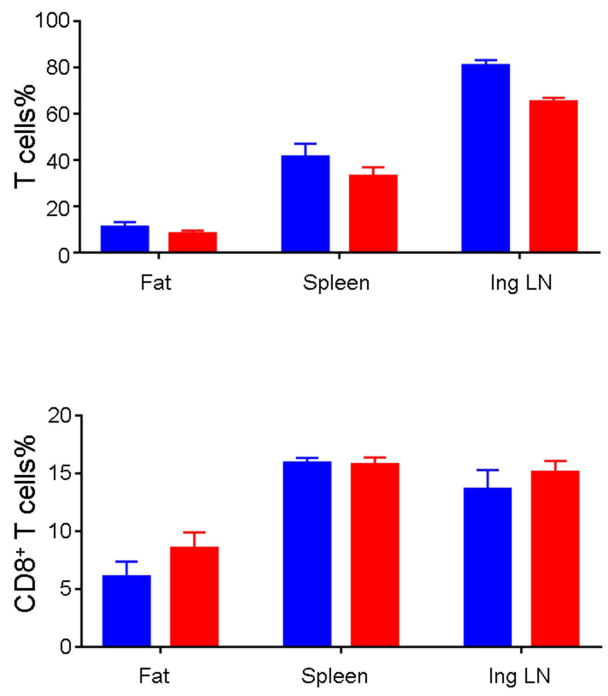

B
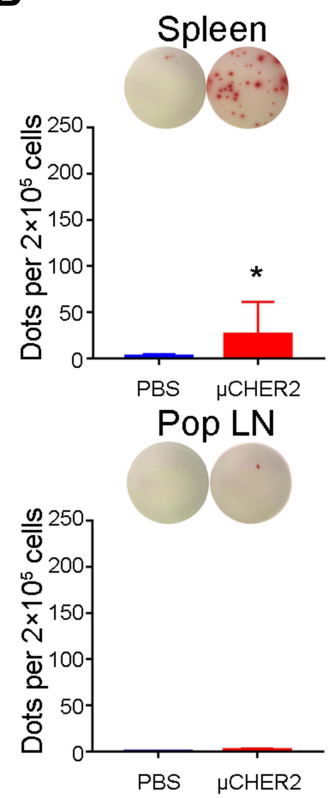

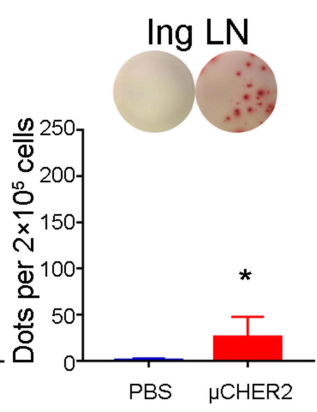

Fat

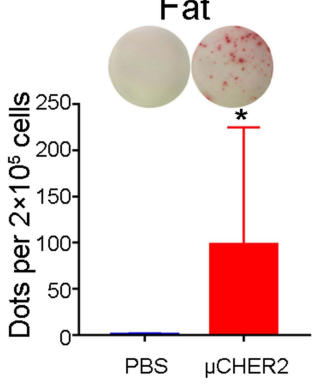

C
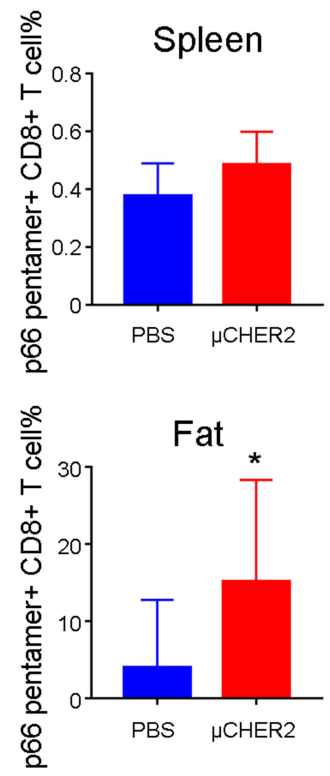

Figure 4 HER2-specific $T$ cell response after $\mu$ CHER2 vaccination. (A) Flow cytometry analysis on total T cells and CD8+ T cells in fat pad, spleen and inguinal lymph node at 7 days post-vaccination via mammary gland fat pad. (B) ELISpot assay of IFN- $\gamma$ secreting antigen-specific T cells from fat pad, spleen and lymph nodes from mice treated with PBS or $\mu$ CHER2. (C) Flow cytometry assay on HER2-specific $T$ cell levels in spleen and fat pad at 7 days post-vaccination. For antigen-specific $T$ cell analysis, data from independent studies were pooled for analysis. Data were presented as mean $\pm \mathrm{SD}$. $*_{\mathrm{p}}<0.05$.

A

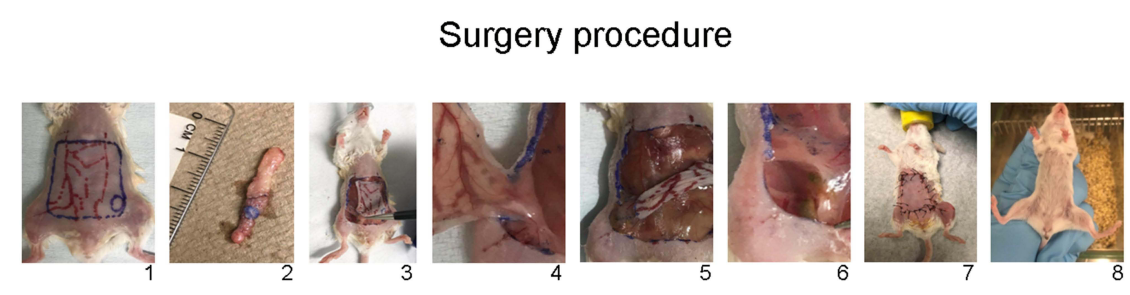

C

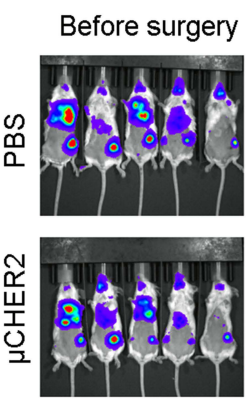

Post surgery
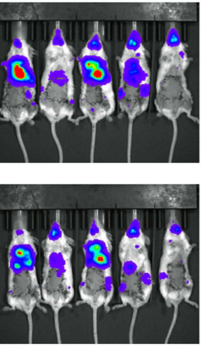

1 week
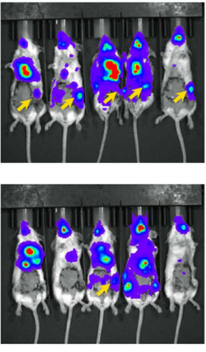

B

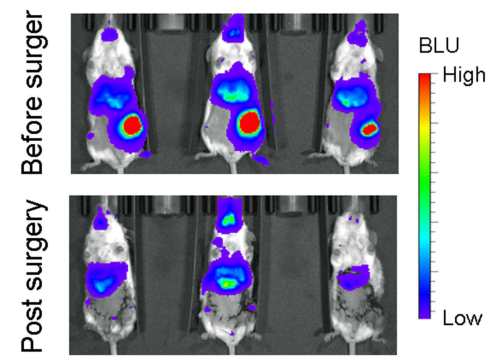

D

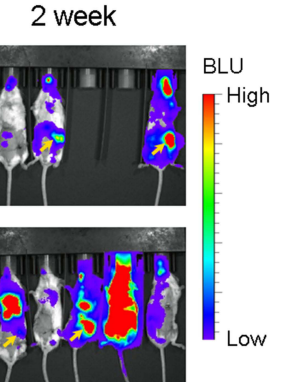

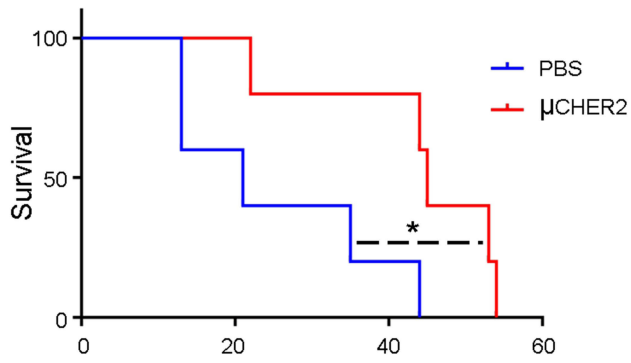

Figure 5 Anti-tumor activity from $\mu \mathrm{CHER} 2$ in mice bearing both orthotopic and systemic metastatic HER2-positive TUBO breast cancer. (A) Procedure of mastectomy and flap surgery on mice. Vaccines were given once via fat tissue flap during surgery. (B) Bioluminescence (BLU) monitoring of tumor resection surgery. (C) In vivo growth inhibition of TUBO tumor by $\mu$ CHER2 treatment via mammary gland fat pad. Systemic TUBO tumor metastasis and local recurrence after mastectomy were monitored with IVIS-200 system ( $n=5$ mice per group). Recurrence of primary tumor is indicated by yellow arrows. (D) Kaplan-Meier plot of animal survival. Difference in survival was compared by log rank test. Data were presented as mean \pm SD. * $<<0.05$. 
the five mice that received flap vaccination got local recurrence at week 1, and the other one at week 2 (Figure 5C, indicated by yellow arrows). Although the orthotopic tumor recurrence was largely inhibited, mice mostly died from bone metastasis in spine. Eventually, mice benefited from receiving $\mu$ CHER2 vaccination on flap, as we observed a significant extension of mice survival in the vaccinated group compared to those in the non-vaccinated group (Figure 5D).

\section{Discussion and Conclusion}

Breast reconstruction after mastectomy using autologous flap is one of the most widely applied methods owing to its longevity and more natural cosmetic results compared to implant-based breast reconstruction. In addition, patients with autologous reconstruction have greater satisfaction with their breasts and greater psychosocial and sexual well-being compared with patients who undergo implant reconstruction. ${ }^{28,29}$ However, surgery can have a negative impact on cancer control. Resection of tumor nodules may cause spread of tumor cells from circulation and stimulate angiogenesis. The surgical process can also compromise the functions of tumor-specific T cells and NK cells, ${ }^{30,31}$ and surgery can induce systemic inflammatory responses and wound healing that promote tumor outgrowth. ${ }^{32}$ Modulation of immunity during and/or after surgery provides a valid approach to solving this problem. For that purpose, we have conducted this preclinical study to test the feasibility of vaccine inoculation in the reconstructed flap. A particulate cancer vaccine loaded with TLR9 agonist and HER2 antigen peptide was tested. Our results have clearly demonstrated the benefit of this approach. By intramammary gland fat pad vaccination following surgery, we have found that $\mu$ CHER 2 activated dendritic cells in flap and draining lymph node to initiate anti-cancer immunity. After 7 days, a strong $\mathrm{T}$ cell stimulation and priming was found, especially in the fat pad, which suggested a potential to prevent local recurrence of breast cancer. Indeed, in animal study, intra-flap vaccination significantly suppressed the residual cancer cells and consequently reduced the incidence of local tumor recurrence on the surgery site, although it only partially attenuated the tumor growth in distal organs.

PSM is an extraordinary vector for cancer vaccine platforms. PSM serves not only as a carrier to deliver antigens, but also an adjuvant to stimulate type I interferon. ${ }^{23}$ Furthermore, DCs can pick up entire PSM vaccine particles and then migrate to draining lymphatic tissues where anti-cancer immunity is initiated. Interestingly, although DOPC liposomal cargos could be released from PSMs slowly, ${ }^{33}$ we found that only the DCs stimulated with PSM vaccine particles could facilitate sufficient immune response. PSM vaccine effectively entered endosomes and activated DCs, followed by stimulation of antigen-specific cytotoxic $\mathrm{CD} 8^{+} \mathrm{T}$ cells against cancer, whereas the immunization of liposome formulation only triggered marginal therapeutic effects. ${ }^{24}$ As microparticles with positive surface charge and numerous pores, PSMs effectively interact with the negatively charged DC surface, and sufficiently feed the DCs with large amount of antigen peptides and adjuvant simultaneously, which is critical for DC maturation, activation and licensing. Due to the limited labeling capacity of PSMs, fluorescent dyes were conjugated with lipid. Although it is still not clear how many liposomes were released from $\mu$ CHER2 after 24 hours (Figure $1 \mathrm{~A}-\mathrm{C}$ ), effective DC stimulation (Figure 3) and $\mathrm{T}$ cell priming in draining lymph node and fat tissue (Figure 4) indicated the sufficient DC stimulation by particulate vaccine $\mu$ CHER 2 .

In our study, infiltration of leukocytes, especially myeloid cells, were found in the fat pad. Although the immunosuppressive nature of myeloid cells may impair cytotoxic $\mathrm{T}$ cell function and favor the tumor progression, ${ }^{34}$ application of correct adjuvant and vaccine carrier can re-polarize myeloidderived suppressor cells (MDSCs) and promote the differentiation into an anti-cancer phenotype. ${ }^{35}$ It is documented that $\mathrm{CpG}$ oligos can block immunosuppression of $\mathrm{MDSCs}^{36,37}$ and, furthermore, the application of PSM particles may stimulate type I interferon expression, ${ }^{23}$ which is required for immune suppressive activity of MDSCs. ${ }^{38}$ So eventually, active antigen-specific $T$ cell successfully inhibited the local recurrence in fat pad. Adipose tissue has a unique immune environment: the adipocytes inside play an important role in obesity-related inflammation. ${ }^{39,40}$ A recent study raised the potential of adipocytes for antigen presentation, ${ }^{41}$ and the contribution of adipose microenvironment and adipocytes in our current strategy may be an important issue that needs to be further addressed.

In conclusion, we have demonstrated that intra-flap vaccination can promote strong anti-cancer immune responses in an animal model of HER2-positive breast cancer.

\section{Acknowledgments}

We thank all the researchers from the Department of Nanomedicine in Houston Methodist Academic Institute, 
for their cooperation and contribution to the experiment. We appreciate the support of members from Department of Breast and Thyroid Surgery.

\section{Funding}

This study was partially supported by internal funding from Houston Methodist and by Special Support Funds of Shenzhen for Introduced High-Level Medical Team (Song Erwei team, SZSM201612010).

\section{Disclosure}

The authors declare no competing financial interests.

\section{References}

1. Siegel RL, Miller KD, Jemal A. Cancer statistics, 2019. CA Cancer J Clin. 2019;69(1):7-34. doi:10.3322/caac.21551

2. Eppenberger-Castori S, Kueng W, Benz C, et al. Prognostic and predictive significance of ErbB-2 breast tumor levels measured by enzyme immunoassay. J Clin Oncol. 2001;19:645-656. doi:10.1200/ JCO.2001.19.3.645

3. Perou CM, Sorlie T, Eisen MB, et al. Molecular portraits of human breast tumours. Nature. 2000;406(6797):747-752. doi:10.1038/ 35021093

4. Gianni L, Eiermann W, Semiglazov V, et al. Neoadjuvant chemotherapy with trastuzumab followed by adjuvant trastuzumab versus neoadjuvant chemotherapy alone, in patients with HER2-positive locally advanced breast cancer (the NOAH trial): a randomised controlled superiority trial with a parallel HER2-negative cohort. Lancet. 2010;375:377-384. doi:10.1016/S0140-6736(09)61964-4

5. Swain SM, Baselga J, Kim SB, et al. Pertuzumab, trastuzumab, and docetaxel in HER2-positive metastatic breast cancer. $N$ Engl J Med. 2015;372:724-734. doi:10.1056/NEJMoa1413513

6. Mohd Sharial MSN, Crown J, Hennessy BT. Overcoming resistance and restoring sensitivity to HER2-targeted therapies in breast cancer. Ann Oncol. 2012;23:3007-3016. doi:10.1093/annonc/mds200

7. Wang J, Tang Y, Jing H, et al. Risk stratification for prediction of locoregional recurrence in patients with pathologic T1-2N0 breast cancer after mastectomy. BMC Cancer. 2020;20:1132. doi:10.1186/ s12885-020-07594-7

8. Melamed R, Rosenne E, Shakhar K, et al. Marginating pulmonary-NK activity and resistance to experimental tumor metastasis: suppression by surgery and the prophylactic use of a beta-adrenergic antagonist and a prostaglandin synthesis inhibitor. Brain Behav Immun. 2005;19:114-126. doi:10.1016/j.bbi.2004.07.004

9. Schmid P, Adams S, Rugo HS, et al. Atezolizumab and Nab-paclitaxel in advanced triple-negative breast cancer. $N$ Engl $J$ Med. 2018;379:2108-2121. doi:10.1056/NEJMoa1809615

10. Sharma P, Allison JP. The future of immune checkpoint therapy. Science. 2015;348(6230):56-61. doi:10.1126/science.aaa8172

11. Miliotou AN, Papadopoulou LC. CAR T-cell therapy: a new era in cancer immunotherapy. Curr Pharm Biotechnol. 2018;19(1):5-18. doi:10.2174/1389201019666180418095526

12. Sutherland SIM, Ju X, Horvath LG, et al. Moving on from sipuleucel-T: new dendritic cell vaccine strategies for prostate cancer. Front Immunol. 2021;12:641307. doi:10.3389/ fimmu.2021.641307

13. Vogelstein B, Papadopoulos N, Velculescu VE, et al. Cancer genome landscapes. Science. 2013;339(6127):1546-1558. doi:10.1126/ science. 1235122
14. Alexandrov LB, Nik-Zainal S, Wedge DC, et al. Signatures of mutational processes in human cancer. Nature. 2013;500(7463):415-421. doi:10.1038/nature12477

15. Kim A, Lee SJ, Kim YK, et al. Programmed death-ligand 1 (PD-L1) expression in tumour cell and tumour infiltrating lymphocytes of HER2-positive breast cancer and its prognostic value. Sci Rep. 2017;7(1):11671. doi:10.1038/s41598-017-11905-7

16. $\mathrm{Xu} \mathrm{J}, \mathrm{Bao} \mathrm{H}, \mathrm{Wu} \mathrm{X}$, et al. Elevated tumor mutation burden and immunogenic activity in patients with hormone receptor-negative or human epidermal growth factor receptor 2-positive breast cancer. Oncol Lett. 2019;18(1):449-455. doi:10.3892/ol.2019.10287

17. Gennari R, Menard S, Fagnoni F, et al. Pilot study of the mechanism of action of preoperative trastuzumab in patients with primary operable breast tumors overexpressing HER2. Clin Cancer Res. 2004;10 (17):5650-5655. doi:10.1158/1078-0432.CCR-04-0225

18. Chamani R, Ranji P, Hadji M, et al. Application of E75 peptide vaccine in breast cancer patients: a systematic review and meta-analysis. Eur J Pharmacol. 2018;831:87-93. doi:10.1016/j. ejphar.2018.05.010

19. Kuan EL, Ivanov S, Bridenbaugh EA, et al. Collecting lymphatic vessel permeability facilitates adipose tissue inflammation and distribution of antigen to lymph node-homing adipose tissue dendritic cells. J Immunol. 2015;194:5200-5210. doi:10.4049/jimmunol.1500221

20. Xiao L, Yang X, Lin Y, et al. Large adipocytes function as antigen-presenting cells to activate $\mathrm{CD} 4(+) \mathrm{T}$ cells via upregulating MHCII in obesity. Int $J$ Obes. 2016;40:112-120. doi:10.1038/ ijo.2015.145

21. Thomalla M, Schmid A, Neumann E, et al. Evidence of an anti-inflammatory toll-like receptor 9 (TLR 9) pathway in adipocytes. $J$ Endocrinol. 2019;240:325-343. doi:10.1530/JOE-180326

22. Karapetyan L, Luke JJ, Davar D. Toll-like receptor 9 agonists in cancer. Onco Targets Ther. 2020;13:10039-10060. doi:10.2147/OTT. S247050

23. Xia X, Mai J, Xu R, et al. Porous silicon microparticle potentiates anti-tumor immunity by enhancing cross-presentation and inducing type I interferon response. Cell Rep. 2015;11:957-966. doi:10.1016/j. celrep.2015.04.009

24. Mai J, Li Z, Xia X, et al. Synergistic activation of antitumor immunity by a particulate therapeutic vaccine. $A d v$ Sci. 2021;8:2100166. doi:10.1002/advs.202100166

25. Womac DJ, Palanisamy AP, Eslick R, et al. Development of a mouse model of abdominal cutaneous flaps for breast reconstruction. PLoS One. 2013;8(1):e52829. doi:10.1371/journal.pone.0052829

26. Shen H, You J, Zhang G, et al. Cooperative, nanoparticle-enabled thermal therapy of breast cancer. Adv Healthc Mater. 2012;1:84-89. doi:10.1002/adhm.201100005

27. Jakubzick CV, Randolph GJ, Henson PM. Monocyte differentiation and antigen-presenting functions. Nat Rev Immunol. 2017;17:349-362. doi:10.1038/nri.2017.28

28. Santosa KB, Qi J, Kim HM, et al. Long-term patient-reported outcomes in postmastectomy breast reconstruction. JAMA Surg. 2018;153(10):891-899. doi:10.1001/jamasurg.2018.1677

29. Pusic AL, Matros E, Fine N, et al. Patient-Reported outcomes 1 year after immediate breast reconstruction: results of the Mastectomy Reconstruction Outcomes Consortium Study. J Clin Oncol. 2017;35 (22):2499-2506. doi:10.1200/JCO.2016.69.9561

30. Tai LH, de Souza CT, Belanger S, et al. Preventing postoperative metastatic disease by inhibiting surgery-induced dysfunction in natural killer cells. Cancer Res. 2013;73:97-107. doi:10.1158/00085472.CAN-12-1993

31. Ananth AA, Tai LH, Lansdell C, et al. Surgical stress abrogates pre-existing protective $\mathrm{T}$ cell mediated anti-tumor immunity leading to postoperative cancer recurrence. PLoS One. 2016;11:e0155947. doi:10.1371/journal.pone. 0155947 
32. Krall JA, Reinhardt F, Mercury OA, et al. The systemic response to surgery triggers the outgrowth of distant immune-controlled tumors in mouse models of dormancy. Sci Transl Med. 2018;10(436). doi:10.1126/scitranslmed.aan3464

33. Tanaka T, Mangala LS, Vivas-Mejia PE, et al. Sustained small interfering RNA delivery by mesoporous silicon particles. Cancer Res. 2010;70:3687-3696. doi:10.1158/0008-5472.CAN-09-3931

34. Ribechini E, Leenen PJ, Lutz MB. Gr-1 antibody induces STAT signaling, macrophage marker expression and abrogation of myeloid-derived suppressor cell activity in BM cells. Eur J Immunol. 2009;39:3538-3551. doi:10.1002/eji.200939530

35. He W, Liang P, Guo G, et al. Re-polarizing Myeloid-derived Suppressor Cells (MDSCs) with cationic polymers for cancer immunotherapy. Sci Rep. 2016;6:24506. doi:10.1038/srep24506

36. Zoglmeier C, Bauer H, Noerenberg D, et al. CpG blocks immunosuppression by myeloid-derived suppressor cells in tumor-bearing mice. Clin Cancer Res. 2011;17:1765-1775. doi:10.1158/10780432.CCR-10-2672
37. Shirota H, Tross D, Klinman DM. CpG oligonucleotides as cancer vaccine adjuvants. Vaccines. 2015;3:390-407. doi:10.3390/ vaccines 3020390

38. Alicea-Torres K, Sanseviero E, Gui J, et al. Immune suppressive activity of myeloid-derived suppressor cells in cancer requires inactivation of the type I interferon pathway. Nat Commun. 2021;12:1717. doi:10.1038/s41467-021-22033-2

39. Soedono S, Cho KW. Adipose tissue dendritic cells: critical regulators of obesity-induced inflammation and insulin resistance. Int $\mathrm{J} \mathrm{Mol}$ Sci. 2021;22(16):8666. doi:10.3390/ijms22168666

40. Woodall MJ, Neumann S, Campbell K, et al. The effects of obesity on anti-cancer immunity and cancer immunotherapy. Cancers. 2020;12(5):1230. doi:10.3390/cancers 12051230

41. Deng T, Lyon CJ, Minze LJ, et al. Class II major histocompatibility complex plays an essential role in obesity-induced adipose inflammation. Cell Metab. 2013;17(3):411-422. doi:10.1016/j. cmet.2013.02.009

\section{Publish your work in this journal}

The International Journal of Nanomedicine is an international, peerreviewed journal focusing on the application of nanotechnology in diagnostics, therapeutics, and drug delivery systems throughout the biomedical field. This journal is indexed on PubMed Central, MedLine, CAS, SciSearch ${ }^{\mathbb{R}}$, Current Contents ${ }^{\mathbb{R}} /$ Clinical Medicine, $^{-}$
Journal Citation Reports/Science Edition, EMBase, Scopus and the Elsevier Bibliographic databases. The manuscript management system is completely online and includes a very quick and fair peer-review system, which is all easy to use. Visit http://www.dovepress.com/ testimonials.php to read real quotes from published authors. 